\title{
COMPLETE MONOTONICITY PROPERTY FOR TWO FUNCTIONS RELATED TO THE $q$-DIGAMMA FUNCTION
}

\author{
Ahmed SAlEm And FARIS AlZahrani
}

Abstract. In this paper, the complete monotonicity property for two functions involving the $q$ digamma function are proven for all positive real $q$ and exploited to establish some sharp inequalities for the $q$-gamma, $q$-digamma and $q$-polygamma functions. Comparisons between our results with previous results are provided.

Mathematics subject classification (2010): 33D05, 26D07, 26A48.

Keywords and phrases: $q$-digamma function, $q$-gamma function, completely monotonic functions, inequalities.

\section{REFERENCES}

[1] C. Krattenthaler, H. M. SRivastava, Summations for basic hypergeometric series involving a q-analogue of the digamma function, Computers Math. Applic., Vol. 32 (2) (1996) 73-91.

[2] A. SAlEM, Some Properties and Expansions Associated with the q-Digamma Function, Quaestiones mathematicae, Vol. 36 (1) (2013) 67-77.

[3] M. E. H. IsmaIl AND M. E. Muldoon, Inequalities and monotonicity properties for gamma and q-gamma functions, in: R. V. M. Zahar (Ed.), Approximation and Computation, International Series of Numerical Mathematics, Vol. 119, Birkhauser, Boston, MA (1994) 309-323.

[4] D. S. MOAK, The q-analogue of Stirling's formula, Rocky Mountain J. Math., Vol. 14 (1984) 403 412.

[5] Z. CHENG, Exact analytical study of ideal Bose atoms in a two-dimensional isotropic harmonic trap, Journal of Statistical Mechanics: Theory and Experiment, Vol. 2017 (6), 063102 (2017).

[6] Z. Cheng, Bose-Einstein condensation of ideal photons in a one-dimensional barrel cavity, Physical Review A, Vol. 93 (2) 023829 (2016).

[7] Z. CHENG, Exact spatial density of ideal Bose atoms in a one-dimensional harmonic trap, Journal of Statistical Mechanics: Theory and Experiment, Vol. 2016 (5) 053102 (2016).

[8] Z. CHENG, Exact thermodynamic theory of an ideal boson gas in a one-dimensional harmonic trap, Journal of Statistical Mechanics: Theory and Experiment, Vol. 2015 (11) P11003 (2015).

[9] Z. CHENG, Exact analytical study of ideal Bose atoms in a one-dimensional harmonic trap, Journal of Statistical Mechanics: Theory and Experiment, Vol. 2015 (9), P09011 (2015).

[10] N. BATIR, Monotonicity properties of q-digamma and q-trigamma functions, Journal of Approximation Theory, Vol. 192 (2015) 336-346.

[11] N. BATIR, q-Extensions of some estimstes associated with the digamma function, J. Approx. Theory 174 (2013) 54-64.

[12] A. SALEM, Sharp bounds for the q-gamma function in terms of the Lambert $W$ function, Ramanujan J., (2018) https: //doi.org/10.1007/s11139-018-0008-9 (In Press).

[13] A. Salem, F. Alzahrani, Improvements of bounds for the q-gamma and the q-polygamma functions, Journal of Mathematical Inequalities, Vol. 11 (3) (2017) 873-883.

[14] A. SAlEM, Completely monotonic functions related to the gamma and the q-gamma functions, Revista de la Real Academia de Ciencias Exactas, Fisicas y Naturales - Serie A: Matematicas, Vol. 111 (1) (2017) 271-280.

[15] A. SALEM, Some classes of completely monotonic functions related to q-gamma and q-digamma functions, Mathematical Inequalities \& Applications, Vol. 19 (3) (2016) 853-862. 
[16] A. SALEM, A certain class of approximations for the q-digamma function, Rocky Mountain Journal of Mathematics, Vol. 46 (5) (2016) 1665-1677.

[17] A. SALEM, Monotonic functions related to the q-gamma function, Monatshefte fur Mathematik, Vol. 179 (2) (2016) 281-292.

[18] A. SAlEm, Some completely monotonic functions associated with the q-gamma and the $q$ polygamma functions, Acta Mathematica Scientia, Vol. 35 (5) (2015) 1214-1224.

[19] A. SAlEM, Completely monotonic functions related to the q-gamma and the q-trigamma functions, Analysis \& Applications, Vol. 13 (2) (2015) 125-134.

[20] A. SAlEM, Complete monotonicity properties of functions involving q-gamma and q-digamma functions, Mathematical Inequalities \& Applications, Vol. 17 (3) (2014) 801-811.

[21] A. SALEM, Two classes of bounds for the q-gamma and the q-digamma functions in terms of the $q$-zeta functions, Banach J. Math. Anal., Vol. 8 (1) (2014) 109-117.

[22] A. SALEM, An infinite class of completely monotonic functions involving the q-gamma function, Journal of Mathematical Analysis and Applications, Vol. 406 (2) (2013) 392-399.

[23] A. SALEM, A completely monotonic function involving q-gamma and q-digamma functions, Journal of Approximation Theory, Vol. 164 (7) (2012) 971-980.

[24] P. GAO, Some Monotonicity Properties of Gamma and q-Gamma Functions, ISRN Mathematical Analysis, Vol. 2011 (2011) 1-15.

[25] H. AlZER, A. Z. Grinshpan, Inequalities for the gamma and q-gamma functions, Journal of Approximation Theory, Vol. 144 (2007) 67-82.

[26] A. Z. GRINSHPAN AND M. E. H. Ismail, Completely monotonic functions involving the gamma and q-gamma functions, Proc. Amer. Math. Soc. Vol. 134 (2006) 1153-1160.

[27] N. Elezović, C. Giordano AND J. PeČARIĆ, Convexity and q-gamma function, Rendiconti del Circolo Matematico di Palermo, Vol. Serie II, Tomo XLVIII (1999) 285-298.

[28] M. E. H. Ismail, L. Lorch And M. E. Muldoon, Completely monotonic functions associated with the gamma function and its $q$-analogues, Journal of Mathematical Analysis and Applications, Vol. 116 (1986) 1-9.

[29] M. Abramowitz, C. A. Stegun, Handbook of Mathematical functions with formulas, Graphs, Mathematical tables 7th printing, Applied Mathematics Series, Vol. 55, Nathional Bureau of standards, Washington, DC, 1964.

[30] N. BATIR, Inequalities for the gamma function, Arch. Math., Vol. 91 (2008) 554-563. 